\title{
ON MODULATED RANDOM MEASURES ${ }^{1}$
}

\author{
JEWGENI H. DSHALALOW \\ Department of Applied Mathematics \\ Florida Institute of Technology \\ Melbourne, FL 32901, USA
}

\begin{abstract}
In this paper the author introduces the notion of a modulated marked random measure, $Z^{\xi}$, on the class of locally compact and $\sigma$-compact spaces with countable bases. As special cases, are marked processes modulated by $\xi$ are considered where $\xi$ is a semi-Markov or semi-regenerative process. For either case, the intensities $\kappa=\lim _{t \rightarrow \infty} \frac{1}{t} E\left[Z^{\xi}([0, t])\right]$ are evaluated in terms of parameters of $\xi$. Examples and applications to inventories, queueing processes and economics are discussed.
\end{abstract}

Key words: random measure, modulated marked random measure, marked process, semi-Markov process, semi-regenerative process.

AMS Subject Classification: Primary 60G57, 60K15, 60K99, secondary $60 \mathrm{~K} 10,60 \mathrm{~K} 25$.

\section{INTRODUCTION}

It is quite common, in practice, that one stochastic process is perturbed by another stochastic process. For instance, a stock market index is occasionally affected by major events in politics. Frequently, these phenomena are ingredients of more complex stochastic systems, like input streams to queueing, inventory, or transportation systems.

In queueing theory there are widely encountered systems with state dependent parameters, i.e. systems where the input or service depends upon the state of the system. The input or service processes may also be perturbed by exterior random factors. Unlike a birth-death type of process, where the input or service, depending on system parameters, changes instantly (which rarely takes place in practice), we assume that parameters of a perturbed process are changed at some random instants of time, when either main perturbations occur or certain decision making is imposed. This is basic to the notion of "modulation."

Many authors (see [8-10]) have considered a doubly stochastic Poisson process $Z^{\xi}$ whose intensity is directed by a Markov process $\xi$ with finitely many states. Such a process is called a Markov modulated Poisson process. In this article, we introduce more general processes, specifically

${ }^{1}$ Received: March, 1991. Revised: August, 1991. 
Poisson processes modulated by semi-Markov or semi-regenerative processes. Such phenomena are encountered in stochastic systems, where input parameters (intensity of interarrivals and batch sizes) are affected by interior random factors, which lead to a more general form of modulation.

One of the target parameters of such a modulated process would be its total intensity, defined as $\kappa=\lim _{t \rightarrow \infty} \frac{1}{t} E\left[Z^{\xi}([0, t])\right]$, giving the total number of events per unit time over an infinite horizon. This is not only of independent interest but it is a key part of many optimization problems involved in controlled stochastic systems (see [1]). In this paper the author also proposes a rigorous construction of a modulated process specifically considering such important implementations as random processes modulated by semi-Markov or semi-regenerative processes. Some examples from queueing theory applications are discussed.

\section{PRELIMINARIES}

In this section we give a brief review of basic notions of the theory of random measures and stochastic processes. For more facts about random measures the reader can be referred to monographs by Daley and Vere-Jones [4] or Karr [7].

Let $E$ be a locally compact and $\sigma$-compact topological space with a countable base, and let $\mathscr{B}=\mathscr{B}(E)$ denote the Borel $\sigma$-algebra.

\subsection{Notation. Denote}

Ho $\sim$ the space of all Radon measures on $\mathfrak{B}$

$\mathcal{H}_{p} \sim$ the subspace of all point (counting) Radon measures on $\mathfrak{B}$

$\mathcal{H}_{a} \sim$ the subspace of all purely atomic Radon measures on $\mathfrak{B}$.

Let $\mathrm{C}_{K}$ denote the space of all continuous functions on $E$ with compact support. Consider the mapping $\Phi_{f}(\mu)=\int f d \mu, \mu \in \mathcal{H}$, for a fixed $f \in \mathrm{C}_{K}$. Let $\mathcal{T}_{\nu}$ denote the vague topology in $\mathcal{H}$ relative to which all these mappings are continuous.

Denote by $\mathfrak{R}=\mathfrak{B}(\mathcal{H})=\sigma\left(\mathcal{T}_{\nu}\right)$ as the Borel $\sigma$-algebra in $\mathcal{H}_{\text {b }}$ and by $\mathfrak{M}_{0}$ as the $\sigma$-algebra of Baire sets in $\mathcal{H}$, i.e. $\mathfrak{M}_{0}$ is generated by all maps $\Phi_{f}$. The corresponding traces of $\sigma$-algebras of all sets in notation 2.1 are denoted by $\mathfrak{M}_{p}=\mathfrak{M} \cap \mathfrak{A}_{p}$, etc.

2.2 Theorem (cf. Bauer [2], corollary 2, p. 206 and theorem, p. 243). Let E be locally compact, $\sigma$-compact and let $E$ have a countable base. Then $\mathfrak{M}_{0}=\mathfrak{M}$.

For what follows, we will assume that we meet the conditions imposed on $E$ in theorem 2.2 so as to have $\mathfrak{M}_{0}=\mathfrak{M}$. 
Let $\{\Omega, F, P\}$ be a probability space. Recall that

(i) a random measure on $E$ is a measurable mapping $M$ of $(\Omega, F)$ into $(\mathcal{H}, \mathfrak{M})$,

(ii) a counting (or point) measure is a measurable mapping $N$ of $(\Omega, \mathscr{F})$ into $\left(\mathcal{H}_{p}, \mathbb{P}_{p}\right)$.

Let $N=\sum_{i=1}^{\infty} \varepsilon_{T_{i}}$ for a sequence $\left\{T_{i}\right\}$ of random instants of times on $\mathbb{R}_{+}$such that $T_{1} \leq T_{2} \leq \ldots$. Then, $N$ is a counting measure or with its more general representation

$$
N=\sum_{i=1}^{K} \varepsilon_{X_{i}}
$$

where $K$ is a random variable valued in $\overline{\mathbf{N}}_{0}=\{0,1, \ldots, \infty\}$. It is also customary to call a counting measure the counting process. For a purely atomic measure $M \in \mathcal{H}_{a}$, we can generalize (2.3) by setting $M=\sum_{i=1}^{K} U_{i} \varepsilon_{X_{i}}$, where $U_{i}$ are nonnegative random variables.

Let $N=\sum \varepsilon_{X_{i}}$ be a counting measure on $E$ and let $E^{\prime}$ be another locally compact and $\sigma$ compact topological space with a countable base. A marked counting measure with underlying counting measure $N$ is any counting measure

$$
\bar{N}=\sum \varepsilon_{\left(X_{i}, U_{i}\right)}
$$

on $E \times E^{\prime}$. The random element $U_{i}$ of $E^{\prime}$ is called the mark associated to $X_{i}$ and $E^{\prime}$ is called the mark space.

Independent marking. Let $N=\sum \varepsilon_{X_{i}}$ be a counting measure on $E$ and let $U_{i}$ be independent and identically distributed random elements of $E^{\prime}$ such that $N$ and $U_{i}$ are independent. Then the marked counting measure $\bar{N}$ is said to be obtained from $N$ by independent marking.

Position-dependent marking. Given a kernel $K$ from $E$ to $E^{\prime}$ we can construct the mark sequence so that the $U_{i}$ are conditionally independent given $N$, with $P\left\{Z_{i} \in A \mid N\right\}=K\left(X_{i}, A\right)$ so that, in addition, the distribution of $U_{i}$ depends only on $X_{i}$.

Let $N=\sum \varepsilon_{X_{i}}$ be a counting measure on $E$ and let $\bar{N}=\sum \varepsilon_{\left(X_{i}, U_{i}\right)}$ be a marked counting measure with mark space $\mathbb{R}_{+}$, obtained from $N$ by "position-dependent marking” by use of a transition kernel $K$. Then the purely atomic random measure, $M=\sum U_{i} \varepsilon_{X_{i}}$, is called a $K$ compound of $N$ or compound counting measure determined by $N$ and $K$.

2.3 Definitions (see Dshalalow [6]).

(i) Let $T$ be a stopping time for the stochastic process $\left\{\Omega, \mathcal{F},\left(P^{x}\right)_{x \in \Psi}, Z(t) ; t \geq 0\right\} \rightarrow(\Psi$, $\mathfrak{B}(\Psi)$ ). $Z$ is said to have the locally strong Markov property at $T$, if for each bounded random variable $\zeta: \Omega \rightarrow \Psi^{r}$ and for each Baire function $f: \Psi^{r} \rightarrow \mathbb{R}, r=1,2, \ldots$, it holds true that

$$
E^{x}\left[f \circ \zeta \circ \theta_{T} \mid \mathscr{F}_{T}\right]=E^{Z_{T}}[f \circ \zeta] P^{x} \text {-a.s. on }\{T<\infty\},
$$

where $\theta_{y}$ is the shift operator.

(ii) A stochastic process $\left\{\Omega, \mathscr{F},\left(P^{x}\right)_{x \in \Psi}, Z(t) ; t \geq 0\right\} \rightarrow(\Psi, \mathfrak{B}(\Psi))$ with $\Psi \preceq \mathbb{N}$ is called 
semi-regenerative if

a) there is a counting measure $N=\sum_{n=1}^{\infty} \varepsilon_{t_{n}}$ on $\mathbb{R}_{+}$such that $t_{n} \rightarrow \infty(n \rightarrow \infty)$ and such that each $t_{n}$ is a stopping time relative to the canonic filtering $\sigma\left(Z_{y} ; y \leq t\right)$,

b) the process $Z$ has the locally strong Markov property at $t_{n}, n=1,2, \ldots$,

c) $\left\{Z\left(t_{n}+0\right), t_{n} ; n=0,1, \ldots\right\}$ is a Markov renewal process.

(iii) Let $\left\{\xi_{n}, t_{n}\right\}$ be an irreducible and aperiodic Markov renewal process with a discrete state space $\Psi$. Denote $\beta_{x}=E^{x}\left[t_{1}\right]$ and $\beta=\left(\beta_{x} ; x \in \Psi\right)^{\mathrm{T}}$. Suppose that the imbedded Markov chain $\xi_{n}$ is ergodic and that $\boldsymbol{P}$ is its invariant probability measure. We call the Markov renewal process recurrent-positive if its mean inter-renewal time, denoted $\boldsymbol{P} \boldsymbol{\beta}$, is finite. An irreducible and aperiodic and recurrent-positive Markov renewal process is called ergodic.

\section{MODULATED RANDOM MEASURES}

Let $E$ be a locally compact and $\sigma$-compact space with a countable base. Let $\mathcal{H}$ be the space of all Radon measures on $\mathscr{B}(E)$, and let $\mathfrak{M}$ be the Borel $\sigma$-algebra in $\mathcal{H}$ generated by the vague topology $\mathcal{T}_{\nu}$.

\subsection{Definitions.}

(i) Let $\{\Omega, \mathscr{T}, P, \xi(t), t \in E\} \rightarrow(\Psi, \mathscr{B}(\Psi))$ be a stochastic process on $E$ and let $\xi_{\omega}$ denote the $\omega$-section of $\xi$. Then, for $\Gamma \in \mathfrak{B}(\Psi)$ and $B \in \mathfrak{B}(E)$, we define

$$
Y_{\Gamma}=B \cap \xi_{\omega}^{-1}(\Gamma)
$$

and call it the holding time of $\xi$ in $\Gamma$ on set $B$.

Clearly, for each fixed $\omega, Y_{\Gamma}$ is a measurable subset of $E$ which can be measured by any Radon measure on $\mathscr{B}(E)$. In general, $Y_{\Gamma}$ is a mapping from $\Omega$ into $\mathscr{B}(E)$ which can be made a random set after we define the $\sigma$-algebra $\mathfrak{B}_{\Gamma}=\left\{\Lambda \subseteq \mathfrak{B}(E): Y_{\Gamma}^{-1}(\Lambda) \in \mathscr{F}\right\}$.

(ii) Assume that there is a countable, measurable decomposition $\sum_{j=0}^{\infty} \Gamma_{j}$ of $\Psi$ (where $\sum$ stands for the union of disjoint sets). Consider a (partial) marked random measure as

with mark space $E^{\prime}$. Introduce

$$
\left.Z^{j}=\sum_{i} \varepsilon_{\left(X_{i}, T_{j}\right.} T_{i} \Gamma_{j}\right)
$$

$$
Z^{\xi}=Z^{\xi}(\omega, B)=\sum_{j=0}^{\infty} Z^{j}\left(Y_{\Gamma_{j}}\right)
$$

Clearly, $Z^{\xi}$ is a random measure from $(\Omega, \mathcal{F})$ into $(\mathcal{H}, \mathfrak{M}) . Z^{\xi}$ is called a marked random measure modulated by the process $\xi$.

Consider some special cases.

[C1] Let $Z^{j}$ be a compound random measure (with mark space $E^{\prime}=\mathbb{R}_{+}$) 


$$
Z^{j}=\sum_{i} X_{i} \Gamma_{j} \varepsilon_{T_{i} \Gamma_{j}}
$$

obtained from the underlying counting measure $N_{j}=\sum_{i} \varepsilon_{T_{i}} \Gamma_{j}$ by independent marking, i.e. for each $\Gamma_{j},\left\{X_{i \Gamma_{j}}\right\}$ is a sequence of independent and identically distributed random variables with common mean denoted $\alpha_{j}$. Assume that $N_{j}$ is a Poisson counting measure with mean measure $\mu_{j} \in \mathcal{M}$.

3.2 Lemma. For the modulated random measure $Z^{\xi}$ under the conditions in [C1] and initial measure $\nu$,

$$
E^{\nu}\left[Z^{\xi}\right]=\sum_{j=0}^{\infty} \alpha_{j} \int_{B} P^{\nu}\left\{\xi \in \Gamma_{j}\right\} d \mu_{j} .
$$

Proof. First, by the monotone convergence theorem we have

$$
E^{\nu}\left[Z^{\xi}\right]=\sum_{j=0}^{\infty} E^{\nu}\left[Z^{j}\left(Y_{\Gamma_{j}}\right)\right]
$$

from which is obtained. Obviously, $\mu_{j}\left(Y_{\Gamma_{j}}\right)$ can be represented as

$$
\mu_{j}\left(Y_{\Gamma_{j}}\right)=\int_{B} I_{\xi_{\omega}}^{-1}\left(\Gamma_{j}\right) d \mu_{j}=\int_{B} I_{\Gamma_{j}} \circ \xi_{\omega} d \mu_{j} .
$$

Now the statement of the lemma follows from (3.2b) and (3.2c) by Fubini's theorem.

3.3 Remark. In order that formula (3.2a) hold true, the random variables $X_{i \Gamma_{j}}$ need not be valued in $\mathbb{R}_{+}$as [C1] suggests. We may weaken this condition assuming that the terms of $\left\{X_{i \Gamma_{j}}\right\}$ are valued in $\mathbb{R}$ and putting appropriate constraints on partial sums of the series in (3.2b), in order to use the generalized monotone convergence theorem (see [5]).

[C2] Let $E=\mathbb{R}$ with its natural topology; let $\mu_{j}$ be absolutely continuous (with respect to the Lebesgue measure l); and let $g_{j}=\frac{d \mu_{j}}{d l}$ be its Radon-Nikodym derivative. Then, from (3.2a) we have

$$
E^{\nu}\left[Z^{\xi}\right]=\sum_{j=0}^{\infty} \alpha_{j} \int_{B} g_{j} P^{\nu}\left\{\xi \in \Gamma_{j}\right\} d l .
$$

In particular, if for each $j$ the Poisson counting measure $N_{j}$ is stationary, i.e. if $g_{j}=\lambda_{j}$ (a positive constant), we have from (3.4)

$$
E^{\nu}\left[Z^{\xi}\right]=\sum_{j=0}^{\infty} \alpha_{j} \lambda_{j} \int_{B} P^{\nu}\left\{\xi \in \Gamma_{j}\right\} d l .
$$

Consider some special cases of modulated random measures.

\section{[C3] SEMI-MARKOV MODULATED RANDOM MEASURE}

Let $E=\mathbb{R}_{+}$and let $N_{j}$ be a stationary orderly Poisson counting measure with intensity $\lambda_{j}$. For each $j,\left\{X_{i j}\right\}$ are supposed to be nonnegative integer-valued random variables with finite means $\alpha_{j}=E\left[X_{i j}\right]$. Let $\left\{\Omega, \mathcal{F},\left(P^{x}\right)_{x \epsilon \Psi}, \xi(t), t \geq 0\right\} \rightarrow \Psi=\{0,1, \ldots\}$ be the minimal semi-Markov process associated with the Markov renewal process $\left\{\Omega, \mathcal{F},\left(P^{x}\right)_{x \in \Psi}, \xi_{n}=\xi\left(t_{n}+0\right), t_{n}\right\} \rightarrow\left(\Psi \times \mathbb{R}_{+}\right.$, 
$\left.\mathscr{B}\left(\Psi \times \mathbb{R}_{+}\right)\right)$. Then, $Z^{\xi}$ in $(3.1 \mathrm{~b})$ is said to be a marked random measure modulated by the semiMarkov process $\xi$.

Let $E^{x}$ denote the expectation with respect to the probability measure $P^{x}$.

3.6 Definition. The number

$$
\kappa=\lim _{t \rightarrow \infty} E^{\alpha}\left[\frac{1}{t} Z^{\xi}([0, t])\right]
$$

is called the total intensity of $Z^{\xi}$ (over infinite horizon), provided that the limit in (3.6a) exists.

3.7 Notation. We assume that the Markov renewal process $\left\{\xi_{n}, t_{n}\right\}$ is ergodic. Let $\boldsymbol{P}$ be the invariant probability measure of the imbedded Markov chain $\left\{\xi_{n}\right\}$. Let $\beta=\left(\beta_{x} ; x \in \Psi\right)^{\mathrm{T}}, \beta_{x}$ $=E^{x}\left[t_{1}\right], \lambda=\left(\lambda_{x} ; x \in \Psi\right)^{\mathrm{T}}$ and $\alpha=\left(\alpha_{x} ; x \in \Psi\right)^{\mathrm{T}}$ and denote $\rho=\alpha * \beta * \lambda$, the Hadamard product of vectors $\alpha, \beta$ and $\lambda$. Let $\Theta^{x}(j, t)=\int_{0}^{t} P^{x}\{\xi(u)=j\} l(d u)$. Denote $\Theta^{x}(t)=\left(\Theta^{x}(j, t) ; j \in \Psi\right)$.

3.8 Lemma (cf. Çinlar [3]). Let $\xi$ be the semi-Markov process associated with an ergodic Markov renewal process $\left\{\xi_{n}, t_{n}\right\}$ and let $P$ be the invariant probability measure of the imbedded Markov chain $\left\{\xi_{n}\right\}$. Then for $x \in E, \lim _{t \rightarrow \infty} \frac{1}{t} \Theta^{x}(t)$ exists; it is independent of $x$ and it is equal to $\frac{P * \beta^{\mathrm{T}}}{P \beta}$.

3.9 Theorem. The total intensity of $Z^{\xi}$ modulated by the semi-Markov process $\xi$ is determined from the formula:

$$
\kappa=\frac{P \rho}{P \beta} .
$$

Proof. The statement follows from (3.5) and lemma 3.8.

\subsection{Examples.}

(i) A trivial special case follows from theorem 3.9, when the process $Z^{\xi}$ is independent of the semi-Markov process $\xi$. The process $Z^{\xi}$ is then a compound Poisson process $Z$ with magnitudes of jumps described by a sequence $\left\{X_{n}\right\}$ of independent and identically distributed random variables with common mean $\alpha$. The associated Poisson point process has intensity $\lambda$. Thus the rate of the process $Z$ should be $\lambda \alpha$. On the other hand, from notation 3.7 , we have $P \rho=\lambda a P \beta$, which yields the same result, $\lambda \alpha$, in the right-hand side of $(3.9 \mathrm{a})$.

(ii) Consider the following queueing system studied in [1]. Let $Q(t)$ denote the number of units in a single-server queueing system at time $t \geq 0$. Let $\xi_{n}=\xi\left(t_{n}+0\right), n=1,2, \ldots$, where $t_{n}$ are successive moments of service completions. We assume here that the server capacity, service time and the input process depend upon the number of units in the system as follows. If at time $t_{n}+$ the number of units $\xi_{n}$ is $j$ the following hold.

- Within the random time interval $\left(t_{n}, t_{n+1}\right)$ the input flow of units is a compound Poisson process with intensity $\lambda_{j}$ and with batch sizes $X_{1 j}, X_{2 j}, \ldots$ as independent and identically distribut- 
ed random variables with the common mean $\alpha_{j}$.

- The server takes for service the $n$th group of units of size $\min \{j, m(j)\}$ (where $m(k)$ is the server capacity, $k=0,1, \ldots)$, provided that $j>0$. If $j=0$, the server begins its idle period which ends as soon as the next group of units arrives at the system. This group is of size $X_{10}$ and the server takes $\min \left\{X_{10}, m(0)\right\}$ units for the next service.

- The $n$th group is served a random time with the distribution function $B_{j} \in\left\{B_{0}, B_{1}, \ldots\right\}$ of general type.

Here $\xi$ is a semi-Markov process imbedded in $Q$ and the process $Z^{\xi}$ describes the bulk input process in the queueing systems with state-dependent parameters (i.e. state-dependent input and service processes). Let $S(B)$ denote the total number of processed customers in time-set $B$ $\in \mathbb{B}\left(\mathbb{R}_{+}\right)$. According to theorem 6.3 of [1], the total intensity of the output process equals $\lim _{t \rightarrow \infty} \frac{E^{x}[S([0, t])]}{t}=\frac{P \rho}{P \beta}$, i.e. the same rate as the input process. Specifically it follows that the expected number of units in the system in equilibrium either is finite or diverges more slowly than with the unit speed. In other words, $\lim _{t \rightarrow \infty} \frac{E^{x}[Q(t)]}{t}=0$ (which is due to the fact that $Q(t)=Q(0)$ $\left.+Z^{\xi}([0, t])-S([0, t])\right)$.

\section{[C4] SEMI-REGENERATIVE MODULATED RANDOM MEASURE}

A compound Poisson process can also be perturbed by a semi-regenerative process on which the Poisson process has little or no affect. A typical example is the influence of the stock market on an individual stock. Assume that in this case a stock market index (for instance the Dow Jones Industrial Average) is varying from time to time, primarily affected by major trades imposed by large mutual funds. In addition, the stock market is perturbed by a sequence $\left\{t_{n}\right\}$ of "catastrophes" or shocks caused by major political or economic events. Actions of mutual funds, of course, depend merely upon the market values at times $t_{n}, n=1,2, \ldots$. Consequently, trades between these times can be described by a semi-Markov modulated Poisson process discussed in [C3]. Truncating values of the stock market index to the next integer, we will deal with a semi-regenerative process $\xi$ relative to the sequence $\left\{t_{n}\right\}$. If the value of an individual stock is dependent on trades and if it depends also upon $\xi$, we can form a semi-regenerative modulated Poisson process, or a nonstationary compound Poisson process with both intensity $\lambda(\xi(t))$ and magnitudes $X_{\cdot \xi(t)}$ dependent on values $\xi(t)$ of the stock market. Other examples of semi-regenerative modulated Poisson processes are inventories affected by price indexes or economic indicators, shot noise processes, and stochastic control processes.

Let $Z^{\xi}$ be a marked random measure modulated by a process $\left\{\Omega, \mathcal{F},\left(P^{x}\right)_{x \epsilon \Psi}, \xi(t) ; t \geq 0\right\}$ $\rightarrow \Psi$, which is supposed to be an ergodic semi-regenerative process (relative to the Markov renewal process $\left.\left\{\xi_{n}, t_{n}\right\}\right)$ with the stationary probability distribution $\pi=\left(\pi_{j} ; j \in \Psi\right)$. 
The following ergodic lemma is due to Çinlar [3] or lemma 6.1 in [1].

3.11 Lemma. Let $\xi$ be an ergodic semi-regenerative process with the stationary probability measure $\boldsymbol{x}$. Then

$$
\lim _{t \rightarrow \infty} \frac{1}{t} \int_{0}^{t} P^{x}\{\xi(u)=j\} l(d u)=\pi_{j} .
$$

3.12 Theorem. The semi-regenerative modulated Poisson process $Z^{\xi}$ has the total intensity

$$
\kappa=\lim _{t \rightarrow \infty} \frac{1}{t} E^{\alpha}\left[Z^{\xi}([0, t])\right]=\pi(\lambda * \alpha) .
$$

Proof. By (3.5) and by lemma 3.11, this assertion follows.

Acknowledgement. The author is thankful to the anonymous referee for his critical comments and suggestions.

\section{REFERENCES}

[1] Abolnikov, L.M., Dshalalow, J. and Dukhovny, A., On some queue length controlled stochastic processes, Journ. Appl. Math. Stoch. Analysis, 3, No. 4, 227-244, 1990.

[2] Bauer, H., Probability Theory and Elements of Measure Theory, Academic Press, 1982.

[3] Çinlar, E., Markov renewal theory, Adv. Appl. Prob., 1, 123-187, 1969.

[4] Daley, D. and Vere-Jones, D., An Introduction to the Theory of Point Processes, Springer-Verlag, 1988.

[5] Dshalalow, J., Real Analysis (in preparation).

[6] Dshalalow, J., A single-server queue with random accumulation level, Journ. Appl. Math. Stoch. Anal., 4, 203-210, 1991.

[7] Karr, A., Point Processes and Their Statistical Inference, Marcel Dekker, 1986.

[8] Knessl, C., Matkowsky, B., Schuss, Z. and Tier, T., A Markov-modulated queue I: stationary distribution, Queueing Systems, 1, 355-374, 1987.

[9] Neuts, M.F., Sumita, U. and Takahashi, Y., Renewal characterization of Markov modulated Poisson processes, Journ. Appl. Math. Simulation, 2, No. 1, 53-70, 1989.

[10] Regterschot, G. and de Smit, J., The queue M/G|1 with Markov modulated arrivals and services, Math. Oper. Res., 11, No. 3, 465-483, 1986. 


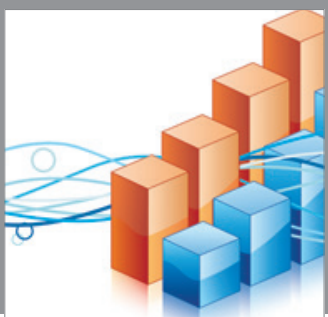

Advances in

Operations Research

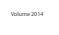

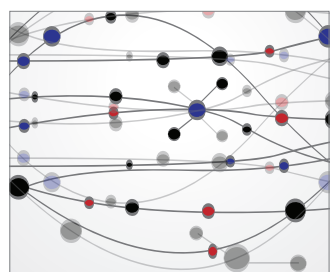

\section{The Scientific} World Journal
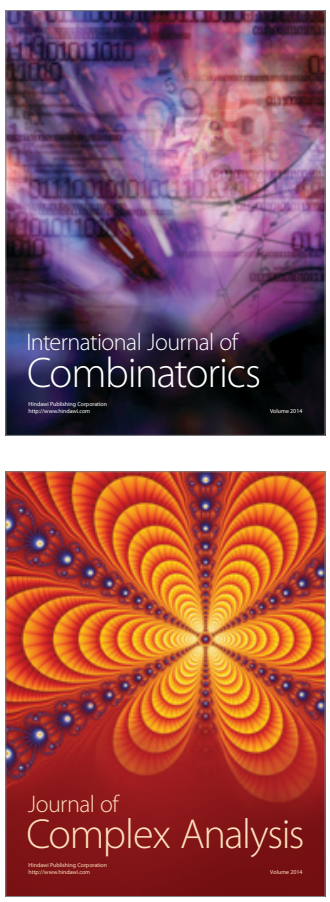

International Journal of

Mathematics and

Mathematical

Sciences
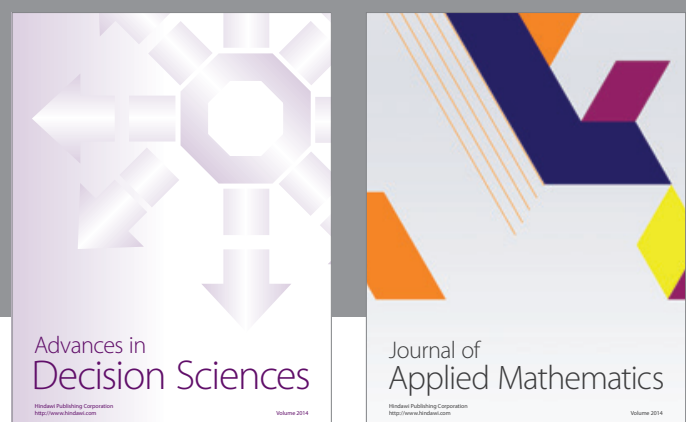

Journal of

Applied Mathematics
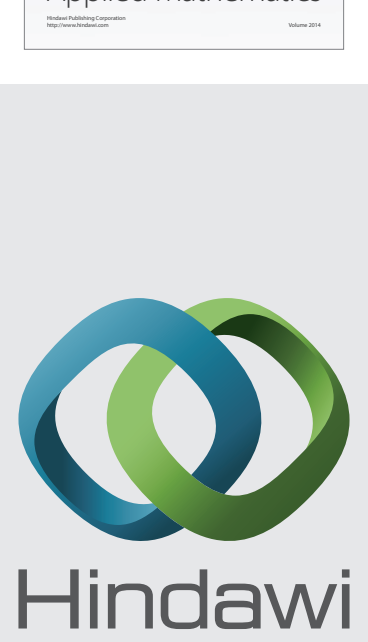

Submit your manuscripts at http://www.hindawi.com
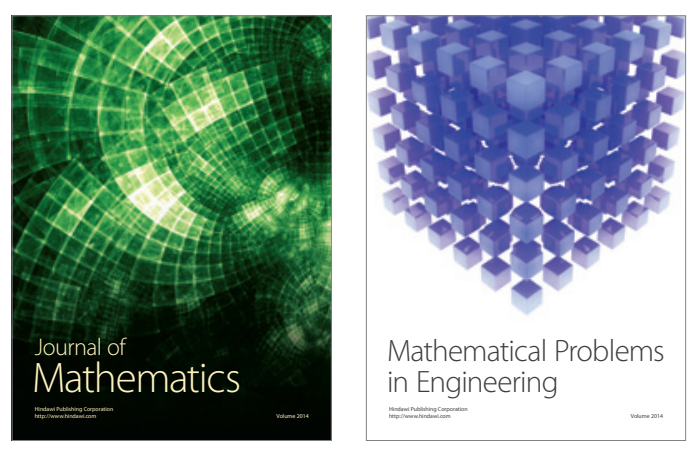

Mathematical Problems in Engineering
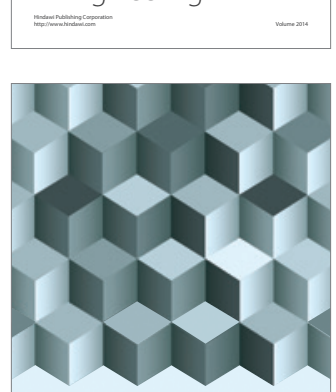

Journal of

Function Spaces
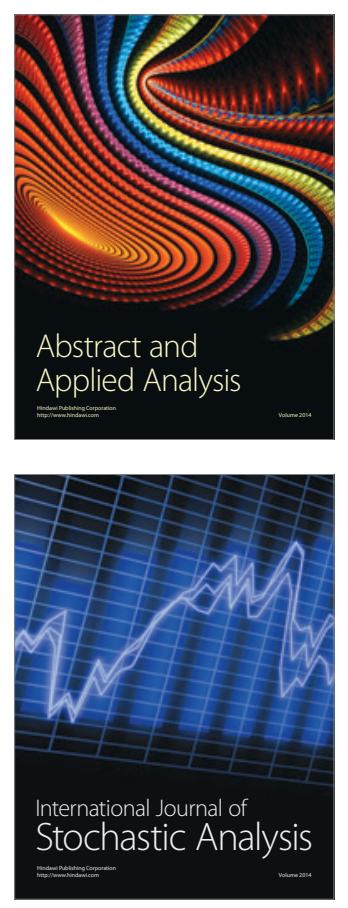

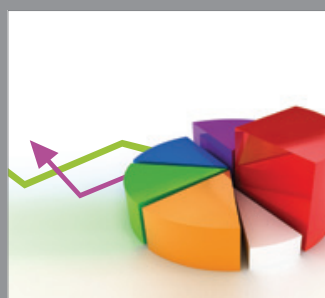

ournal of

Probability and Statistics

Promensencen
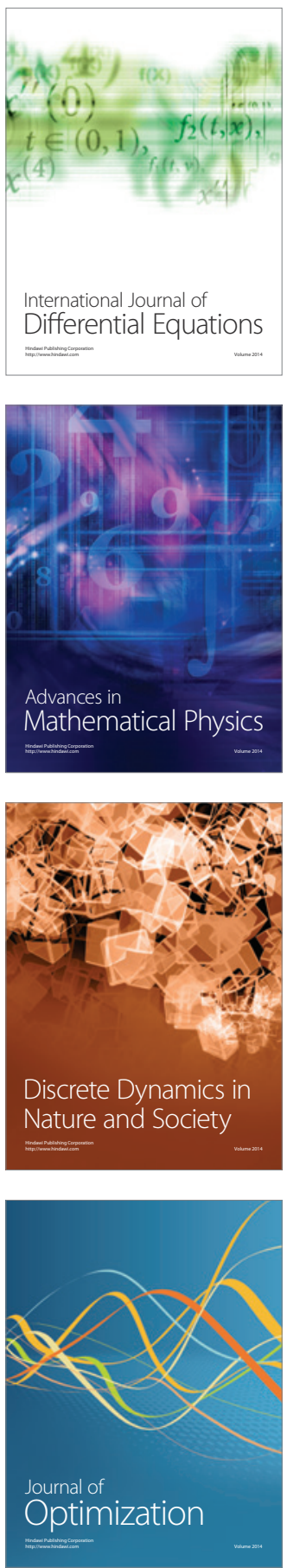